\title{
FET PET reveals considerable spatial differences of tumour burden compared to conventional MRI in newly diagnosed glioblastoma
}

\author{
P. Lohmann 1 , P. Stavrinou², K. Lipke ${ }^{1}$, E.K. Bauer 3 , G. Ceccon ${ }^{3}$, J.-M. Werner ${ }^{3}$, \\ B. Neumaier ${ }^{1}$, G.R. Fink ${ }^{1,3}$, N.J. Shah ${ }^{1,4}$, K.-J. Langen ${ }^{1,5}$, and N. Galldiks ${ }^{1,3,6}$ \\ ${ }^{1}$ Institute of Neuroscience and Medicine (INM-3, -4, -5), Forschungszentrum Juelich, \\ Juelich, Germany \\ ${ }^{2}$ Department of Neurosurgery, University of Cologne, Cologne, Germany \\ ${ }^{3}$ Department of Neurology, University of Cologne, Cologne, Germany \\ ${ }^{4}$ Department of Neurology, University Hospital RWTH Aachen, Aachen, Germany \\ ${ }^{5}$ Department of Nuclear Medicine, University Hospital RWTH Aachen, Aachen, \\ Germany
}

${ }^{6}$ Center of Integrated Oncology (CIO), Universities of Cologne and Bonn, Cologne, Germany

\section{ACKNOWLEDGMENTS}

The authors thank Suzanne Schaden, Trude Plum, Natalie Judov, Silke Frensch, Kornelia Frey, and Lutz Tellmann for assistance in the patient studies; Johannes Ermert, Silke Grafmüller, Erika Wabbals and Sascha Rehbein for radiosynthesis of FET.

\section{Correspondence}

Dr. Philipp Lohmann

Forschungszentrum Juelich

Institute of Neuroscience and Medicine (INM-4)

52425 Juelich

Germany

Phone: +49-2461-61-96357

FAX: +49-2461-61-2302

Email: p.lohmann@fz-juelich.de 


\section{ABSTRACT}

Purpose: Contrast enhancement (CE) in MRI is usually the target for resection or radiotherapy target volume definition in glioblastomas. However, the solid tumour mass may extend beyond areas of CE. Amino acid PET can detect such tumour parts that show no CE. We systematically investigated tumour volumes delineated by amino acid PET and MRI in newly diagnosed, untreated glioblastoma patients.

Methods: Preoperatively, 50 patients with neuropathologically confirmed glioblastoma underwent O-(2-[18 F]-fluoroethyl)-L-tyrosine (FET) PET, fluid-attenuated inversion recovery (FLAIR) and contrast-enhanced MRI. Areas of CE were manually segmented. FET PET tumour volumes were segmented using a tumour-to-brain ratio $\geq 1.6$. The percentage of overlapping volumes (OV), Dice and Jaccard spatial similarity coefficients (DSC; JSC) were calculated. FLAIR images were evaluated visually.

Results: In $86 \%$ of patients ( $n=43$ ), the FET tumour volume was significantly larger than the volume of CE $(21.5 \pm 14.3 \mathrm{~mL}$ vs. $9.4 \pm 11.3 \mathrm{~mL} ; P<0.001)$. Forty patients $(80 \%)$ showed both an increased uptake of FET and CE. In these 40 patients, the spatial similarity between FET and CE was low (mean DSC, $0.39 \pm 0.21$; mean JSC, $0.26 \pm 0.16)$. Ten patients (20\%) showed no CE, and one of these patients showed no FET uptake. In $10 \%$ of patients $(n=5)$, increased FET uptake was present outside of areas of FLAIR hyperintensity.

Conclusions: Our data show that the metabolically active tumour volume delineated by FET PET is significantly larger than tumour volume delineated by CE. Furthermore, the data strongly suggest that the information derived from both imaging modalities should be integrated into the management of newly diagnosed glioblastoma patients. 


\section{KEYWORDS}

FET PET tumour volume; volumetry; amino acid PET; MRI contrast enhancement;

FLAIR hyperintensity; target volume definition 


\section{INTRODUCTION}

Despite significant advances in diagnostics and therapy over the last decades, the prognosis for patients with glioblastoma (GBM), the most frequent form of glioma and the most aggressive and lethal primary brain tumour, remains dismal (median survival, 15-20 months) [1-3]. Currently, the proposed first-line therapy for patients with GBM is a maximally safe resection followed by radiotherapy plus concomitant and adjuvant temozolomide chemotherapy $[4,1]$. Importantly, the extent of the tumour resection is associated with an improved overall survival, i.e., a "complete" resection - currently defined as a lack of contrast enhancement in the early postoperative MRI within 24-48 $\mathrm{h}$ after surgery [5] - is superior to resection smaller than the area of CE or a biopsy only [6-8]. Consequently, contrast-enhancing tumour portions on MRI constitute the target of neurosurgical resection in the majority of cases $[9,4]$. Similar to neurosurgical resection planning, radiotherapy target volume definition is also often based on contrast-enhanced MRI [10]. In stark contrast, several studies revealed that a considerable amount of glioma cells or even the main tumour burden may not show contrast enhancement, particularly in patients with GBM [11-13]. Furthermore, a histopathological comparison of whole-brain sections of brain tumour patients with MRI data showed that the extent of malignant gliomas is not correctly delineated by conventional MRI [14]. Finally, the precise delineation of glioma tissue is particularly problematic if no contrast enhancement is present as is frequently observed in lowgrade gliomas and even $30-40 \%$ of anaplastic gliomas show no contrast enhancement [15]. In rare cases, this also occurs in patients with GBM $[16,17]$. In these cases, the hyperintensity on T2-weighted or fluid-attenuated inversion recovery (FLAIR) MRI is frequently used for treatment planning [18]. 
Over the past years, several studies have shown that PET using radiolabelled amino acids detects non-contrast-enhancing tumour parts and offers a better delineation of gliomas before treatment planning [19-22]. A number of amino acid PET studies using L-methyl-[11 $\mathrm{C}]$-methionine (MET) or O-(2-[18 $\mathrm{F}]-$ fluoroethyl)-L-tyrosine (FET) correlated imaging findings with histology. The results suggest a reliable detection of glioma tissue independent of the integrity of the blood-brain barrier [23, 19, 24, 25]. Consistent with these findings, a post-mortem study compared MET PET and contrast-enhanced CT findings with whole-brain histopathology of a patient with an anaplastic astrocytoma and revealed that MET PET was able to correctly identify the full tumour extent while the contrast-enhanced CT missed more than $50 \%$ of the tumour mass [26].

To the best of our knowledge, it remains to be investigated in which fraction of untreated glioblastomas significant differences between contrast enhancement, FLAIR hyperintensity and FET accumulation occur and whether this is relevant in clinical practice. To this end, we performed a systematic volumetric comparison of FET uptake with MR contrast enhancement and a visual comparison with FLAIR hyperintensity in a series of 50 patients with newly diagnosed GBM concerning size, overlap, and spatial correlation. 


\section{PATIENTS AND METHODS}

\section{Patients}

Between February 2010 and August 2017, 50 adult patients with a newly-diagnosed and untreated GBM investigated using FET PET at the Institute of Neuroscience and Medicine, Forschungszentrum Juelich, Germany, were included in this retrospective study (female, 19; male 31; mean age, $56 \pm 14$ years; range, 26-79 years). Postoperatively, a neuropathological work-up confirmed a GBM in all patients. The study adheres to the standards established in the declaration of Helsinki. All patients had provided written informed consent before each FET PET investigation. Given its retrospective nature, the local ethics committee of the University of Aachen waived the requirement for additional approval. Table 1 presents further details of all patients.

\section{MR Imaging}

Before neuropathological confirmation, all patients underwent a three-dimensional T2weighted FLAIR and a three-dimensional T1-weighted MRI scan after intravenous administration of gadoteric acid $(0.1 \mathrm{mmol} / \mathrm{kg}$ body weight). Five patients were investigated on a 3T Achieva MRI scanner (Philips Medical Systems, Inc.), 17 patients on a 1.5T Intera MRI scanner (Philips Medical Systems, Inc.), and 28 patients on a high-resolution 3T hybrid PET/MR scanner (3T Tim TRIO, Siemens Medical Systems, Inc.). FLAIR images were not available or incomplete in four patients.

\section{FET PET Imaging}

The amino acid FET was produced and applied as described previously [27, 28]. Before neuropathological confirmation and in close temporal proximity to the MRI examination, all patients underwent a dynamic PET scan from 0 to 50 min postinjection (p.i.) of $3 \mathrm{MBq}$ of FET per kg body weight. The time interval between MRI and 
PET was in the range of $0-20$ days (median, 0 days). Fifteen patients were measured on a stand-alone PET scanner (ECAT EXACT HR+, Siemens Medical Systems, Inc.) in 3D mode (32 rings, axial field of view, $15.5 \mathrm{~cm}$ ). The reconstructed dynamic dataset consisted of 16 time-frames ( $5 \times 1 \mathrm{~min} ; 5 \times 3 \mathrm{~min} ; 6 \times 5 \mathrm{~min})$. A transmission scan (duration, $10 \mathrm{~min})$ using three rotating line sources $\left({ }^{68} \mathrm{Ge} /{ }^{68} \mathrm{Ga}\right)$ was used for attenuation correction. Before iterative OSEM reconstruction (16 subsets, 6 iterations), data were corrected for dead time, random and scattered coincidences. Thirty-five patients were scanned on a high-resolution 3T hybrid PET/MR scanner (BrainPET, Siemens Medical Systems, Inc., 72 rings, axial field of view, $19.2 \mathrm{~cm}$ ). Image data were corrected for random and scatter coincidences, as well as dead time before OP-OSEM reconstruction provided by the manufacturer (2 subsets, 32 iterations). The reconstructed dynamic data set consisted of 16 time-frames $(5 \times 1 \mathrm{~min} ; 5 \times 3 \mathrm{~min} ; 6 \times$ 5 min). Since the hybrid PET/MR scanner does not provide a transmission source, attenuation correction was performed by a template-based approach using MRI [29].

\section{Data Analysis and Calculation of Tumour Volumes}

After adjusting the spatial resolution of the BrainPET images to those of the ECAT EXACT HR+ scanner by applying a $2.5 \mathrm{~mm}$ 3D Gaussian filter [30], PET images were corrected for motion, and the MR and PET images (summation images from 20 to 40 min p.i.) were resliced to a voxel size of $1 \times 1 \times 1 \mathrm{~mm}^{3}$ using the software PMOD (version 3.505, PMOD Technologies Ltd.) to provide a robust co-registration and an accurate volume calculation. Afterwards, MR and PET images were co-registered using rigid matching algorithms from PMOD. The results from the co-registration process were validated visually and, if necessary, manually corrected according to anatomic landmarks. Areas of contrast enhancement in the T1-weighted MRI were manually segmented on each consecutive transverse slice, and the sum of the 
circumscribed areas was multiplied by the slice thickness to obtain volumes-of-interest (VOI) representing the total volume of contrast-enhancement for each patient. Necrotic parts were excluded from the volume of contrast-enhancement. A second investigator validated the results from co-registration and the manual delineation. Since most patients exhibit diffuse and widespread FLAIR hyperintensities, a reproducible and objective segmentation was not possible.

The standardized uptake value (SUV) was used for normalization of the FET uptake by dividing the radioactivity in the tissue by the radioactivity injected per gram of body weight. A spherical VOI of constant size (diameter, $30 \mathrm{~mm}$ ) was positioned in normal appearing brain tissue including grey and white matter in the hemisphere contralateral to the lesion. A three-dimensional auto-contouring process using a tumour-to-brain ratio (TBR) of 1.6 or more was used for segmentation of the tumour volume. This threshold is based on a biopsy-controlled study in which this value separated best between vital tumour and healthy brain parenchyma in FET PET [19]. Mean TBR

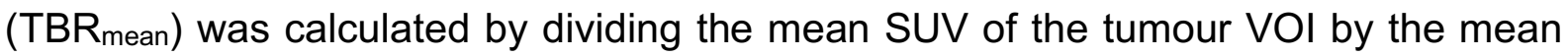
SUV of the background VOI. We calculated the maximum TBR (TBR $\max$ ) by dividing the maximum SUV of the tumour VOI by the mean SUV of the background VOI.

\section{Calculation of Spatial Correlation and Overlap of MRI- and PET-based Tumour}

\section{Volumes}

As a measure of spatial correlation [31], the spatial similarity between the VOls defined by contrast enhancement on MRI and increased tracer uptake in FET PET was evaluated using the Dice similarity coefficient (DSC) [32] and the Jaccard similarity coefficient (JSC) [33]. The DSC is defined as 


$$
\operatorname{DSC}\left(\mathrm{V}_{\mathrm{PET}}, \mathrm{V}_{\mathrm{MRI}}\right)=\frac{2\left(\mathrm{~V}_{\mathrm{PET}} \cap \mathrm{V}_{\mathrm{MRI}}\right)}{\mathrm{V}_{\mathrm{PET}}+\mathrm{V}_{\mathrm{MRI}}}
$$

$V_{P E T}, V_{M R I}$ are the VOls defined by FET PET and MRI, respectively. The JSC is calculated according to

$$
\operatorname{JSC}\left(\mathrm{V}_{\mathrm{PET}}, \mathrm{V}_{\mathrm{MRI}}\right)=\frac{\mathrm{V}_{\mathrm{PET}} \cap \mathrm{V}_{\mathrm{MRI}}}{\mathrm{V}_{\mathrm{PET}} \cup \mathrm{V}_{\mathrm{MRI}}}=\frac{\mathrm{DSC}}{2-\mathrm{DSC}},
$$

which is the ratio of the intersection and the union of the FET PET and the MRI VOI. Additionally, the overlap volume (OV) [31] was calculated and considers, in contrast to the DSC and JSC, volume differences of the FET PET and MRI VOls. The OV is defined as the ratio of the intersection and the smallest volume according to

$$
\mathrm{OV}\left(\mathrm{V}_{\mathrm{PET}}, \mathrm{V}_{\mathrm{MRI}}\right)=\frac{\mathrm{V}_{\mathrm{PET}} \cap \mathrm{V}_{\mathrm{MRI}}}{\min \left(\mathrm{V}_{\mathrm{PET}}, \mathrm{V}_{\mathrm{MRI}}\right)}
$$

While DSC and JSC are similarity coefficients that range between 0 (no similarity) and 1 (perfect agreement), the OV is an overlap coefficient and a value of $100 \%$ indicates that one volume is completely contained within the other. All calculations were implemented and performed in MATLAB (Version R2015a, Mathworks, Inc.). Figure 1 illustrates the differences between the three coefficients.

\section{Visual Analysis of Spatial Correlation of PET- and FLAIR-based Tumour Volumes}

The spatial correlation of FET- and FLAIR-based tumour volumes was assessed visually according to the following criteria: i) FET-based tumour volume larger than FLAIR-based tumour volume; ii) FET-based tumour volume smaller than FLAIR-based tumour volume; iii) FET-based tumour volume similar to FLAIR-based tumour volume; 
and iv) FET-based tumour volume partially located outside of FLAIR-based tumour volume. The results from the visual analysis were validated by a second investigator.

\section{Statistical Analysis}

Descriptive statistics are provided as mean and standard deviation or median and range. The non-parametric Wilcoxon rank-sum test was used for intergroup comparison. $p$-values of less than 0.05 were considered significant. Statistical analysis was performed using the statistical software packages SPSS (version 24, IBM Corp.) and Excel (Excel for Mac 2016, version 16.12, Microsoft Corp.). 


\section{RESULTS}

\section{Tumour Volumes}

One patient showed neither FET uptake nor contrast-enhancement. All of the remaining 49 patients showed an increased uptake of FET (averaged TBR mean, $2.2 \pm$ 0.4 ; range, 1.2 - 3.3; averaged TBR $\max , 4.2 \pm 1.4$; range, 2.1 - 8.7). Ten patients (20\%) showed no contrast enhancement on MRI (see Figure 2). The FET PET tumour volumes and the volumes of contrast enhancement were in a range of $0.0-56.4 \mathrm{~mL}$ and $0.0-44.6 \mathrm{~mL}$, respectively. In $86 \%$ of the patients $(n=43)$, the average FET PET tumour volume was significantly larger than the average volume of contrast enhancement $(21.5 \pm 14.3 \mathrm{~mL}$ vs. $9.4 \pm 11.3 \mathrm{~mL} ; P<0.001)$. Table 1 and Figure 3 summarize these findings.

\section{Overlap and Spatial Similarity of MRI- and PET-based Tumour Volumes}

Forty of 50 patients $(80 \%)$ showed both an increased FET uptake and contrast enhancement in MRI. In these 40 patients, the OV was moderate (mean OV, $77 \% \pm$ $26 \%$; range, $8 \%-100 \%$ ), but the spatial similarity was low (mean DSC, $0.39 \pm 0.21$; range, $0.02-0.70$; mean JSC, $0.26 \pm 0.16$; range, $0.01-0.54$ ). Furthermore, 6 of these 40 patients (15\%) showed both a low OV of less than $50 \%$ (mean OV, $28 \pm 15 \%$ ) as well as a low spatial similarity (mean DSC, $0.15 \pm 0.08$; mean JSC, $0.08 \pm 0.05$ ). In total, $32 \%$ of patients $(n=16)$ showed either no contrast enhancement (see Figure 2) or an OV $<50 \%$ in combination with a low spatial similarity (DSC $<0.4$; JSC $<0.3$ ).

\section{Overlap and Spatial Similarity Depending on Tumour Volumes}

A subgroup analysis revealed that 6 of 50 patients (12\%) with a FET PET tumour volume smaller than the volume of contrast enhancement showed both a low OV 
(mean OV, $40 \pm 21 \%$; range, $8-71 \%$ ) and low spatial similarity (mean DSC, $0.33 \pm$ 0.24 ; range $0.05-0.70$; mean JSC, $0.22 \pm 0.19$; range, $0.02-0.54)$. Patients with a FET PET tumour volume larger than the contrast enhancement $(n=43)$ had a high OV (mean OV, $84 \% \pm 20 \%$; range, 24 - 100\%) but a low spatial similarity (mean DSC, 0.40 \pm 0.24 ; range $0.02-0.69$; mean JSC, $0.27 \pm 0.18$; range, $0.01-0.53$ ). Table 1 and Figure 3 summarize the results. Figure 4 shows two representative patients.

\section{Spatial Correlation of PET- and FLAIR-based Tumour Volumes}

The FET PET tumour volume was clearly smaller than the FLAIR volume in $70 \%$ of the patients $(n=35)$. In 10 patients $(20 \%)$, the FET PET volume was similar to the FLAIR volume and one patient (2\%) had a FET PET volume that was larger than the FLAIR signal. Five patients $(10 \%)$ had a considerable portion of FET PET tumour volume outside of the area of FLAIR hyperintensity (Figure 2, top row). The results are summarized in Table 1. 


\section{DISCUSSION}

Using MR and amino acid PET, various previous studies compared predominantly tumour volume sizes. To the best of our knowledge, this study is the first in this group of patients which assesses and evaluates various volumetric parameters. The main finding of our study is that there are significant differences concerning size, overlap, and the spatial correlation, indicating that conventional contrast-enhanced MRI underestimates substantially the tumour volume. In 43 of 50 patients ( $86 \%$ ) the FET PET tumour volume was significantly more extensive than the volume of contrast enhancement. Interestingly, 10 of these 50 patients $(20 \%)$ even showed no contrast enhancement. One of these patients additionally showed no FET uptake. Furthermore, in the remaining 40 patients (80\%) with an increased FET uptake and contrast enhancement, the spatial similarity (evaluated using the DSC and JSC) was low despite a moderate OV (average OV, 77\%). Moreover, in 6 of these 40 patients (15\%), the spatial similarity between FET PET and contrast enhancement was low and also the OV was below $50 \%$, i.e., relevant areas of FET uptake were located partially outside and hence exceeded beyond the area of contrast enhancement (see Figure 4). In total, 16 of 50 patients (32\%) showed either no contrast enhancement at all or a low OV in combination with a low spatial similarity between FET and contrast enhancement. Note that the measure OV alone needs to be interpreted with caution, i.e., a high OV only represents two spatially congruent volumes if both volumes are of similar size. If the volumes have different sizes, the interpretation of the OV alone may be misleading. The addition of the measures of spatial similarity such as the DSC and the JSC for a combined interpretation of the results leads to a correct impression of the spatial orientation (Figure 1, Figure 4). In the present study, the OV of nearly $80 \%$ between FET PET and contrast-enhanced MRI in our patient cohort might lead to the premature conclusion that contrast-enhanced MRI adequately represents the 
metabolically active tumour in the majority of cases. However, the combined evaluation of OV and the low spatial similarity measures (DSC $<0.4$; JSC $<0.3$ ) reveals that the high $\mathrm{OV}$ is biased by the initial volumetric differences between the tumour volumes defined by contrast enhancement and increased FET uptake. These important differences between the used volumetric measures are illustrated in more detail in Figures 1 and 4 .

The visual analysis of the spatial differences between FLAIR images and FET PET tumour volumes revealed significant spatial differences. Although the hyperintense areas on FLAIR images were larger than both contrast enhancement and increased FET PET in $70 \%$ of patients, a considerable portion of FET tumour volume was located outside of the FLAIR signal in $10 \%$ of the patients. Thus, even the combination of contrast enhancement and FLAIR hyperintensity does not cover the full extent of the tumour in all glioblastoma patients (Figure 2, top row).

Using the PET amino acid tracer MET, several studies investigated the role of the amino acid PET in brain tumour assessment in comparison with conventional MRI. For example, Pirotte and colleagues [34] revealed that a MET PET-guided "complete" tumour resection prolonged significantly the overall survival of patients with high-grade gliomas whereas a "complete" resection based upon the contrast enhancement did not. Grosu and colleagues [35] investigated the value of MET PET relative to conventional MRI for target volume definition before radiotherapy in resected highgrade glioma patients. In $74 \%$ of the patients, gross tumour volumes based on MET PET were considerably more extensive than those based on contrast-enhanced MRI, a finding subsequently confirmed by Mahasittiwat and colleagues [36]. Assessing the spatial differences between MET PET and contrast-enhanced MRI in patients with 
recurrent GBM, we observed that contrast-enhanced MRI substantially underestimated the metabolically active tumour volume as defined by MET PET [24]. However, the short half-life of ${ }^{11} \mathrm{C}$ (20 minutes) limits the use of MET to institutions with an onsite cyclotron.

Since the ${ }^{18} \mathrm{~F}$-labeled amino acid tracer FET (half-life, 110 minutes) overcomes the logistic disadvantages of MET, FET PET has been intensively evaluated over the last two decades for the detection of vital tumour tissue for biopsy guidance and treatment planning, especially neurosurgical resection or radiotherapy [37]. Usually, summed images from 20 to 40 min p.i. are used for the analysis of FET PET in brain tumours. Unterrainer and colleagues [38] recently reported that the FET PET tumour volume is considerably larger in early summation images from 5 to 15 min p.i., especially in high grade gliomas. Consequently, the true discrepancy between contrast-enhanced MRI and FET PET might be even larger if early summation images are used for FET PET tumour segmentation. However, in that study a validation with histology derived from local stereotactic biopsies for the assessment of the true tumour extent was not performed.

Predominantly, the impact of FET PET on radiotherapy target volume definition in patients with low- and high-grade gliomas has been investigated [39-42]. These studies provide converging evidence that the spatial correlation of MRI- and FET PETbased target volumes is low and that FET PET target volumes are considerably more extensive than MR-defined target volumes. Consequently, the integration of FET PET for target volume definition has been recommended. However, these studies did not further investigate in detail the overlapping volumes or the spatial correlation using dedicated quantitative measures such as the DSC or JSC. To the best of our 
knowledge, only two studies investigated the spatial similarity of FET PET and contrast enhancement using the DSC, analogous to our study. Henriksen and colleagues [43] calculated the DSC between FET PET and contrast-enhanced MRI for 17 of 32 patients with pre-treated low- and high-grade gliomas. The estimated spatial similarity was low (mean DSC, 0.19 ) and only 8 of the 17 patients had a DSC of more than 0.1 . In our study, the spatial similarity was higher (mean DSC, 0.39) and only five patients had a DSC below 0.1 , most likely due to a more homogenous patient collective. Henriksen and colleagues also reported that the metabolic tumour volume as delineated by a TBR of more than 1.6 (i.e., identical to our approach) was significantly larger than the volume of contrast enhancement, which is in line with our results. However, the patient number in that study is small, and the patient sample comparatively heterogeneous (5 patients with low-grade glioma and 12 patients with high-grade glioma), which is also reflected in a variety of pre-treatments including antiangiogenic therapy.

Unterrainer and colleagues [44] also calculated the DSC between FET PET and contrast-enhanced MRI in 20 patients with high-grade gliomas at initial diagnosis or recurrence prior to any further therapy. The mean DSC was 0.38 , which is in good agreement with our results (mean DSC, 0.39).

Considering that a maximally safe tumour resection is a critical treatment component for GBM patients due to its survival benefits, the importance of an adequate preoperative volumetric representation of the metabolically active tumour tissue becomes especially apparent. There is substantial evidence in the literature that the extent of tumour resection constitutes a prognostic factor in glioma patients $[45,9,8$, 4]. Consequently, a correct preoperative spatial delineation of the vital tumour tissue is essential for maximizing the extent of resection. In many centres, 5-aminolevulinic 
acid-derived fluorescence (5-ALA) is intraoperatively used to identify tumour tissue [4]. It has been demonstrated that a 5-ALA fluorescence-guided tumour resection in patients with GBM is associated with an improved progression-free survival at 6 months [4]. In that study, it could also be demonstrated that 5-ALA identifies tumour tissue beyond areas of preoperative MRI contrast enhancement indicating that 5-ALA delineates non-enhancing tumour portions. This confirms the low sensitivity of MRI to correctly represent tumour tissue [46]. Note that amino acid PET using FET was found to be even more sensitive to detect glioma tissue than 5-ALA fluorescence [47].

A limitation of our study is the lack of spatial evaluation of neuroimaging findings by histology derived from local stereotactic biopsies. Thus, prospective studies including spatial correlation of imaging findings with histology obtained by stereotactic biopsy are needed to confirm our results. On the other hand, the patient cohort is fairly homogenous, i.e., in all patients, a GBM was confirmed neuropathologically according to the 2016 WHO classification of brain tumours [48], and all tumours were untreated. Furthermore, three different quantitative measures to describe the spatial similarity and OV were used to assess volumetric and spatial differences between FET PET and contrast enhancement. Furthermore, spatial differences between FLAIR hyperintensities and FET PET tumour volumes were visually evaluated.

In conclusion, our data suggest that the metabolically active tumour volume in patients with newly diagnosed, untreated GBM is critically underestimated by contrastenhanced MRI. Furthermore, the spatial similarity between the contrast-enhanced MRI and FET PET is low, i.e., areas of FET uptake extend substantially beyond the area of contrast enhancement and also beyond the area of FLAIR hyperintensity in some cases. In these cases, FET PET yields essential information for a correct tumour 
delineation beyond and thereby contributes significantly to tailoring the tumour resection. In the future, it has to be demonstrated that a FET PET-guided tumour resection or target volume delineation before radiotherapy significantly impacts upon the patient's survival. Currently, a randomized phase-II trial (NOA-10 / GLIAA study) [49] evaluates whether a radiotherapy target volume delineation based on amino acid PET in comparison with contrast-enhanced MRI prolongs significantly survival in patients with progressive GBM. 


\section{COMPLIANCE WITH ETHICAL STANDARDS}

Funding: This study was funded by Wilhelm Sander-Stiftung, Munich, Germany (grant number 2016.069.1 to N.G.)

Conflict of Interest: The authors declare that they have no conflict of interest.

Ethical approval: All procedures performed in studies involving human participants were in accordance with the ethical standards of the institutional and/or national research committee and with the 1964 Helsinki declaration and its later amendments or comparable ethical standards. For this type of study formal consent is not required.

Informed consent: Informed consent was obtained from all individual participants included in the study. 


\section{REFERENCES}

1. Stupp R, Mason WP, van den Bent MJ, Weller M, Fisher B, Taphoorn MJ et al. Radiotherapy plus concomitant and adjuvant temozolomide for glioblastoma. N Engl J Med. 2005;352(10):987-96.

2. Chinot OL, Wick W, Mason W, Henriksson R, Saran F, Nishikawa R et al. Bevacizumab plus radiotherapy-temozolomide for newly diagnosed glioblastoma. $\mathrm{N}$ Engl J Med. 2014;370(8):709-22.

3. Weller M, Butowski N, Tran DD, Recht LD, Lim M, Hirte H et al. Rindopepimut with temozolomide for patients with newly diagnosed, EGFRvIII-expressing glioblastoma (ACT IV): a randomised, double-blind, international phase 3 trial. Lancet Oncol. 2017;18(10):1373-85.

4. Stummer W, Pichlmeier U, Meinel T, Wiestler OD, Zanella F, Reulen HJ et al. Fluorescence-guided surgery with 5-aminolevulinic acid for resection of malignant glioma: a randomised controlled multicentre phase III trial. Lancet Oncol. 2006;7(5):392-401.

5. Albert FK, Forsting M, Sartor K, Adams HP, Kunze S. Early postoperative magnetic resonance imaging after resection of malignant glioma: objective evaluation of residual tumor and its influence on regrowth and prognosis. Neurosurgery. 1994;34(1):45-60. 
6. Aghi MK, Nahed BV, Sloan AE, Ryken TC, Kalkanis SN, Olson JJ. The role of surgery in the management of patients with diffuse low grade glioma: A systematic review and evidence-based clinical practice guideline. J Neurooncol. 2015;125(3):50330.

7. Brown TJ, Brennan MC, Li M, Church EW, Brandmeir NJ, Rakszawski KL et al. Association of the Extent of Resection With Survival in Glioblastoma: A Systematic Review and Meta-analysis. JAMA Oncol. 2016;2(11):1460-9.

8. Kreth FW, Thon N, Simon M, Westphal M, Schackert G, Nikkhah G et al. Gross total but not incomplete resection of glioblastoma prolongs survival in the era of radiochemotherapy. Ann Oncol. 2013;24(12):3117-23.

9. Lacroix M, Abi-Said D, Fourney DR, Gokaslan ZL, Shi W, DeMonte F et al. A multivariate analysis of 416 patients with glioblastoma multiforme: prognosis, extent of resection, and survival. J Neurosurg. 2001;95(2):190-8.

10. Niyazi M, Brada M, Chalmers AJ, Combs SE, Erridge SC, Fiorentino A et al. ESTRO-ACROP guideline "target delineation of glioblastomas". Radiother Oncol. 2016;118(1):35-42.

11. Halperin EC, Bentel G, Heinz ER, Burger PC. Radiation therapy treatment planning in supratentorial glioblastoma multiforme: an analysis based on post mortem topographic anatomy with CT correlations. Int J Radiat Oncol Biol Phys. 1989;17(6):1347-50. 
12. Lunsford LD, Martinez AJ, Latchaw RE. Magnetic resonance imaging does not define tumor boundaries. Acta Radiol Suppl. 1986;369:154-6.

13. Eidel O, Burth S, Neumann JO, Kieslich PJ, Sahm F, Jungk C et al. Tumor Infiltration in Enhancing and Non-Enhancing Parts of Glioblastoma: A Correlation with Histopathology. PLoS One. 2017;12(1):e0169292.

14. Tovi M, Hartman M, Lilja A, Ericsson A. Mr Imaging in Cerebral Gliomas. Acta Radiol. 1994;35(5):495-505.

15. Ginsberg LE, Fuller GN, Hashmi M, Leeds NE, Schomer DF. The significance of lack of MR contrast enhancement of supratentorial brain tumors in adults: histopathological evaluation of a series. Surg Neurol. 1998;49(4):436-40.

16. Rapp M, Heinzel A, Galldiks N, Stoffels G, Felsberg J, Ewelt C et al. Diagnostic performance of 18F-FET PET in newly diagnosed cerebral lesions suggestive of glioma. J Nucl Med. 2013;54(2):229-35.

17. Hutterer M, Nowosielski M, Putzer D, Jansen NL, Seiz M, Schocke M et al. [18F]fluoro-ethyl-I-tyrosine PET: A valuable diagnostic tool in neuro-oncology, but not all that glitters is glioma. Neuro Oncol. 2013;15(3):341-51.

18. Weller M, van den Bent M, Tonn JC, Stupp R, Preusser M, Cohen-Jonathan-Moyal E et al. European Association for Neuro-Oncology (EANO) guideline on the diagnosis and treatment of adult astrocytic and oligodendroglial gliomas. The Lancet Oncology. 2017;18(6):e315-e29. 
19. Pauleit D, Floeth F, Hamacher K, Riemenschneider MJ, Reifenberger G, Muller HW et al. O-(2-[18F]fluoroethyl)-L-tyrosine PET combined with MRI improves the diagnostic assessment of cerebral gliomas. Brain. 2005;128(Pt 3):678-87.

20. Galldiks N, Stoffels G, Filss C, Rapp M, Blau T, Tscherpel C et al. The use of dynamic O-(2-18F-fluoroethyl)-I-tyrosine PET in the diagnosis of patients with progressive and recurrent glioma. Neuro Oncol. 2015;17(9):1293-300.

21. Jansen NL, Suchorska B, Wenter V, Schmid-Tannwald C, Todica A, Eigenbrod S et al. Prognostic significance of dynamic 18F-FET PET in newly diagnosed astrocytic high-grade glioma. J Nucl Med. 2015;56(1):9-15.

22. Albert NL, Weller M, Suchorska B, Galldiks N, Soffietti R, Kim MM et al. Response Assessment in Neuro-Oncology working group and European Association for NeuroOncology recommendations for the clinical use of PET imaging in gliomas. Neuro Oncol. 2016;18(9):1199-208.

23. Pirotte B, Goldman S, Massager N, David P, Wikler D, Vandesteene A et al. Comparison of 18F-FDG and 11C-methionine for PET-guided stereotactic brain biopsy of gliomas. J Nucl Med. 2004;45(8):1293-8.

24. Galldiks N, Ullrich R, Schroeter M, Fink GR, Jacobs AH, Kracht LW. Volumetry of [(11)C]-methionine PET uptake and MRI contrast enhancement in patients with recurrent glioblastoma multiforme. Eur J Nucl Med Mol Imaging. 2010;37(1):84-92. 
25. Mosskin M, Ericson K, Hindmarsh T, Vonholst H, Collins VP, Bergstrom M et al. Positron Emission Tomography Compared with Magnetic-Resonance Imaging and Computed-Tomography in Supratentorial Gliomas Using Multiple Stereotactic Biopsies as Reference. Acta Radiol. 1989;30(3):225-32.

26. Bergström M, Collins VP, Ehrin E, Ericson K, Eriksson L, Greitz T et al. Discrepancies in brain tumor extent as shown by computed tomography and positron emission tomography using [68Ga]EDTA, [11C]glucose, and [11C]methionine. J Comput Assist Tomogr. 1983;7(6):1062-6.

27. Hamacher $\mathrm{K}$, Coenen $\mathrm{HH}$. Efficient routine production of the $18 \mathrm{~F}$-labelled amino acid O-2-18F fluoroethyl-L-tyrosine. Appl Radiat Isot. 2002;57(6):853-6.

28. Langen KJ, Bartenstein P, Boecker H, Brust P, Coenen HH, Drzezga A et al. [German guidelines for brain tumour imaging by PET and SPECT using labelled amino acids]. Nuklearmedizin. 2011;50(4):167-73.

29. Kops ER, Herzog H. Template based attenuation correction for PET in MR-PET scanners. IEEE Nuclear Science Symposium Conference Record. 2008:3786-9.

30. Lohmann P, Herzog H, Rota Kops E, Stoffels G, Judov N, Filss C et al. Dual-timepoint O-(2-[(18)F]fluoroethyl)-L-tyrosine PET for grading of cerebral gliomas. Eur Radiol. 2015;25(10):3017-24. 
31. Besemer AE, Titz B, Grudzinski JJ, Weichert JP, Kuo JS, Robins HI et al. Impact of PET and MRI threshold-based tumor volume segmentation on patient-specific targeted radionuclide therapy dosimetry using CLR1404. Phys Med Biol. 2017;62(15):6008-25.

32. Dice LR. Measures of the Amount of Ecologic Association Between Species. Ecology. 1945;26(3):297-302.

33. Jaccard P. The distribution of the flora in the alpine zone. New Phytol. $1912 ; 11(2): 37-50$

34. Pirotte BJ, Levivier M, Goldman S, Massager N, Wikler D, Dewitte O et al. Positron emission tomography-guided volumetric resection of supratentorial high-grade gliomas: a survival analysis in 66 consecutive patients. Neurosurgery. 2009;64(3):47181.

35. Grosu AL, Weber WA, Riedel E, Jeremic B, Nieder C, Franz M et al. L-(methyl11C) methionine positron emission tomography for target delineation in resected highgrade gliomas before radiotherapy. Int J Radiat Oncol Biol Phys. 2005;63(1):64-74.

36. Mahasittiwat P, Mizoe JE, Hasegawa A, Ishikawa H, Yoshikawa K, Mizuno H et al. I-[METHYL-(11)C] methionine positron emission tomography for target delineation in malignant gliomas: impact on results of carbon ion radiotherapy. Int J Radiat Oncol Biol Phys. 2008;70(2):515-22. 
37. Misch M, Guggemos A, Driever PH, Koch A, Grosse F, Steffen IG et al. (18)F-FETPET guided surgical biopsy and resection in children and adolescence with brain tumors. Childs Nerv Syst. 2015;31(2):261-7.

38. Unterrainer M, Winkelmann I, Suchorska B, Giese A, Wenter V, Kreth FW et al. Biological tumour volumes of gliomas in early and standard 20-40 min (18)F-FET PET images differ according to IDH mutation status. Eur J Nucl Med Mol Imaging. 2018;45(7):1242-9.

39. Rieken S, Habermehl D, Giesel FL, Hoffmann C, Burger U, Rief H et al. Analysis of FET-PET imaging for target volume definition in patients with gliomas treated with conformal radiotherapy. Radiother Oncol. 2013;109(3):487-92.

40. Niyazi M, Geisler J, Siefert A, Schwarz SB, Ganswindt U, Garny S et al. FET-PET for malignant glioma treatment planning. Radiother Oncol. 2011;99(1):44-8.

41. Jaymanne DT, Kaushal S, Chan D, Schembri G, Brazier D, Bailey D et al. Utilizing 18F-fluoroethyl-I-tyrosine positron emission tomography in high grade glioma for radiation treatment planning in patients with contraindications to MRI. J Med Imaging Radiat Oncol. 2018;62(1):122-7.

42. Debus C, Waltenberger M, Floca R, Afshar-Oromieh A, Bougatf N, Adeberg S et al. Impact of (18)F-FET PET on Target Volume Definition and Tumor Progression of Recurrent High Grade Glioma Treated with Carbon-Ion Radiotherapy. Sci Rep. 2018;8(1):7201. 
43. Henriksen OM, Larsen VA, Muhic A, Hansen AE, Larsson HB, Poulsen HS et al. Simultaneous evaluation of brain tumour metabolism, structure and blood volume using [(18)F]-fluoroethyltyrosine (FET) PET/MRI: feasibility, agreement and initial experience. Eur J Nucl Med Mol Imaging. 2016;43(1):103-12.

44. Unterrainer M, Fleischmann DF, Diekmann C, Vomacka L, Lindner S, Vettermann F et al. Comparison of (18)F-GE-180 and dynamic (18)F-FET PET in high grade glioma: a double-tracer pilot study. Eur J Nucl Med Mol Imaging. 2018.

45. Sanai N, Polley MY, McDermott MW, Parsa AT, Berger MS. An extent of resection threshold for newly diagnosed glioblastomas. J Neurosurg. 2011;115(1):3-8.

46. Schucht P, Knittel S, Slotboom J, Seidel K, Murek M, Jilch A et al. 5-ALA complete resections go beyond MR contrast enhancement: shift corrected volumetric analysis of the extent of resection in surgery for glioblastoma. Acta Neurochir (Wien). 2014;156(2):305-12.

47. Floeth FW, Sabel M, Ewelt C, Stummer W, Felsberg J, Reifenberger G et al. Comparison of (18)F-FET PET and 5-ALA fluorescence in cerebral gliomas. Eur J Nucl Med Mol Imaging. 2011;38(4):731-41.

48. Louis DN, Perry A, Reifenberger G, von Deimling A, Figarella-Branger D, Cavenee WK et al. The 2016 World Health Organization Classification of Tumors of the Central Nervous System: a summary. Acta Neuropatho. 2016;131(6):1-18. 
49. Oehlke O, Mix M, Graf E, Schimek-Jasch T, Nestle U, Gotz I et al. Amino-acid PET versus MRI guided re-irradiation in patients with recurrent glioblastoma multiforme (GLIAA) - protocol of a randomized phase II trial (NOA 10/ARO 2013-1). BMC Cancer. 2016;16(1):769. 


\section{TABLES AND FIGURES}

Table 1 Demographic and clinical data of patient cohort and summary of results

\begin{tabular}{|c|c|c|c|c|c|c|c|c|c|c|c|c|c|c|c|c|}
\hline$\#$ & Age & Sex & Histology & Treatment & $\begin{array}{l}\text { Time between } \\
\text { PET and MRI }\end{array}$ & $\begin{array}{l}\mathrm{V}_{\mathrm{FET}} \\
{[\mathrm{mL}]}\end{array}$ & $\begin{array}{c}\mathbf{V}_{\mathrm{CE}} \\
{[\mathrm{mL}]}\end{array}$ & DSC & JSC & ov & $\begin{array}{l}\text { FET vs. } \\
\text { FLAIR }\end{array}$ & $\mathrm{TBR}_{\text {mean }}$ & TBR $_{\max }$ & EoR & IDH genotype & $\begin{array}{c}\text { MGMT promoter } \\
\text { methylation }\end{array}$ \\
\hline 1 & 75 & $\mathrm{~m}$ & GBM & none & 0 & 43.4 & 44.6 & 0.51 & 0.35 & $52 \%$ & $a$ & 1.9 & 3.3 & PR & wt & unmethylated \\
\hline 2 & 71 & $f$ & GBM & none & 14 & 40.5 & 28.1 & 0.47 & 0.31 & $57 \%$ & $a, d$ & 2.0 & 3.8 & PR & wt & methylated \\
\hline 3 & 58 & $\mathrm{~m}$ & GBM & none & 0 & 32.8 & 16.5 & 0.65 & 0.48 & $96 \%$ & a & 2.3 & 4.4 & PR & wt & methylated \\
\hline 4 & 78 & $\mathrm{~m}$ & GBM & none & 1 & 14.0 & 21.9 & 0.16 & 0.09 & $20 \%$ & $a, d$ & 1.8 & 2.6 & $\mathrm{CR}$ & wt & unmethylated \\
\hline 5 & 59 & $\mathrm{~m}$ & GBM & none & 0 & 16.9 & 6.9 & 0.56 & 0.39 & $97 \%$ & $\mathrm{~b}$ & 2.7 & 5.7 & PR & wt & unmethylated \\
\hline 6 & 42 & $f$ & GBM & none & 0 & 31.0 & 11.3 & 0.45 & 0.29 & $82 \%$ & $a$ & 1.9 & 3.7 & $\mathrm{PR}$ & wt & unmethylated \\
\hline 7 & 32 & $f$ & GBM & none & 9 & 6.1 & 2.1 & 0.47 & 0.31 & $94 \%$ & $a$ & 2.8 & 8.7 & $\mathrm{CR}$ & wt & methylated \\
\hline 8 & 66 & $\mathrm{~m}$ & GBM & none & 10 & 28.7 & 29.5 & 0.70 & 0.54 & $71 \%$ & $\mathrm{~b}$ & 2.2 & 4.0 & PR & wt & unmethylated \\
\hline 9 & 50 & $\mathrm{~m}$ & GBM & none & 0 & 21.4 & 6.0 & 0.39 & 0.24 & $89 \%$ & $a$ & 2.2 & 4.0 & $\mathrm{CR}$ & wt & unmethylated \\
\hline 10 & 73 & $f$ & GBM & none & 0 & 49.6 & 25.2 & 0.65 & 0.48 & $97 \%$ & $a$ & 3.3 & 8.3 & $\mathrm{CR}$ & wt & unmethylated \\
\hline 11 & 67 & $f$ & GBM & none & 6 & 28.4 & 10.9 & 0.46 & 0.30 & $82 \%$ & a & 2.1 & 3.3 & $\mathrm{CR}$ & wt & unmethylated \\
\hline 12 & 31 & $\mathrm{~m}$ & GBM & none & 0 & 34.9 & 15.8 & 0.22 & 0.13 & $36 \%$ & $a$ & 2.0 & 3.6 & $\mathrm{CR}$ & mut & methylated \\
\hline 13 & 64 & $f$ & GBM & none & 7 & 47.6 & 41.9 & 0.22 & 0.13 & $24 \%$ & $a$ & 2.0 & 3.8 & PR & wt & methylated \\
\hline 14 & 68 & $\mathrm{~m}$ & GBM & none & 0 & 30.4 & 15.2 & 0.58 & 0.41 & $87 \%$ & $a$ & 2.5 & 4.9 & $\mathrm{CR}$ & n.a. & methylated \\
\hline 15 & 54 & $\mathrm{~m}$ & GBM & none & 0 & 21.1 & 10.6 & 0.65 & 0.48 & $96 \%$ & a & 2.5 & 4.5 & $\mathrm{CR}$ & wt & unmethylated \\
\hline 16 & 50 & $f$ & GBM & none & 0 & 40.7 & 19.0 & 0.60 & 0.43 & $94 \%$ & $b$ & 2.6 & 5.6 & $\mathrm{CR}$ & wt & unmethylated \\
\hline 17 & 40 & $\mathrm{~m}$ & GBM & none & 0 & 41.3 & 14.7 & 0.28 & 0.16 & $53 \%$ & $\mathrm{~b}$ & 2.4 & 5.4 & $\mathrm{CR}$ & mut & methylated \\
\hline 18 & 75 & $\mathrm{~m}$ & GBM & none & 7 & 17.2 & 6.6 & 0.41 & 0.26 & $75 \%$ & - & 2.3 & 4.6 & $\mathrm{CR}$ & wt & unmethylated \\
\hline 19 & 49 & $\mathrm{~m}$ & GBM & none & 17 & 15.1 & 0.9 & 0.11 & 0.06 & $0 \%$ & - & 2.8 & 6.8 & $\mathrm{CR}$ & wt & methylated \\
\hline 20 & 56 & $f$ & GBM & none & 0 & 30.3 & 12.6 & 0.58 & 0.40 & $98 \%$ & $a$ & 2.6 & 4.6 & PR & mut & methylated \\
\hline 21 & 60 & $\mathrm{~m}$ & GBM & none & 11 & 15.4 & 3.3 & 0.35 & 0.21 & $0 \%$ & $\mathrm{~b}$ & 2.5 & 4.1 & $\mathrm{CR}$ & wt & unmethylated \\
\hline 22 & 79 & $\mathrm{~m}$ & GBM & none & 0 & 6.4 & 14.2 & 0.05 & 0.02 & $8 \%$ & a & 1.2 & 2.8 & PR & n.a. & unmethylated \\
\hline 23 & 75 & $\mathrm{~m}$ & GBM & none & 4 & 13.3 & 5.1 & 0.52 & 0.35 & $93 \%$ & a & 2.5 & 4.7 & PR & wt & unmethylated \\
\hline 24 & 45 & $\mathrm{~m}$ & GBM & none & 20 & 25.4 & 9.8 & 0.55 & 0.38 & $98 \%$ & $a$ & 3.1 & 6.4 & $\mathrm{CR}$ & wt & unmethylated \\
\hline 25 & 34 & $\mathrm{~m}$ & GBM & none & 0 & 40.3 & 18.3 & 0.51 & 0.34 & $81 \%$ & $a$ & 2.0 & 3.3 & $\mathrm{CR}$ & n.a. & methylated \\
\hline 26 & 54 & $\mathrm{~m}$ & GBM & none & 0 & 2.3 & 0.4 & 0.31 & 0.18 & $96 \%$ & $a$ & 1.9 & 2.9 & $\mathrm{CR}$ & wt & methylated \\
\hline 27 & 48 & $f$ & GBM & none & 0 & 22.4 & 5.6 & 0.38 & 0.24 & $95 \%$ & $a$ & 2.5 & 5.1 & PR & wt & unmethylated \\
\hline 28 & 67 & $f$ & GBM & none & 0 & 14.3 & 0.5 & 0.06 & 0.03 & $89 \%$ & $b$ & 2.3 & 4.1 & $\mathrm{CR}$ & n.a. & unmethylated \\
\hline 29 & 41 & $f$ & GBM & none & 14 & 31.0 & 0.6 & 0.02 & 0.01 & $44 \%$ & $a$ & 2.0 & 3.2 & B & n.a. & methylated \\
\hline 30 & 50 & $f$ & GBM & none & 10 & 2.8 & 0.0 & - & - & - & $\mathrm{a}$ & 2.2 & 3.3 & $\mathrm{CR}$ & wt & methylated \\
\hline 31 & 66 & $f$ & GBM & none & 30 & 28.1 & 0.0 & - & - & - & $a$ & 1.9 & 3.1 & B & wt & methylated \\
\hline 32 & 33 & $f$ & GBM & none & 6 & 15.2 & 0.2 & 0.02 & 0.01 & $0 \%$ & - & 2.5 & 5.2 & $\mathrm{CR}$ & wt & unmethylated \\
\hline 33 & 66 & $\mathrm{~m}$ & GBM & none & 7 & 10.0 & 1.7 & 0.27 & 0.16 & $94 \%$ & $a$ & 2.2 & 3.3 & B & wt & methylated \\
\hline 34 & 50 & $\mathrm{~m}$ & GBM & none & 0 & 5.1 & 11.4 & 0.21 & 0.12 & $34 \%$ & $\mathrm{a}$ & 1.7 & 2.7 & B & n.a. & methylated \\
\hline 35 & 39 & $\mathrm{~m}$ & GBM & none & 0 & 25.0 & 0.9 & 0.07 & 0.04 & $97 \%$ & $c, d$ & 2.2 & 5.2 & PR & wt & unmethylated \\
\hline 36 & 55 & $\mathrm{~m}$ & GBM & none & 0 & 26.9 & 7.1 & 0.41 & 0.26 & $95 \%$ & $a$ & 2.5 & 5.2 & PR & wt & unmethylated \\
\hline 37 & 51 & $f$ & GBM & none & 11 & 7.5 & 0.0 & - & - & - & $b$ & 2.1 & 2.9 & $\mathrm{CR}$ & mut & methylated \\
\hline 38 & 59 & $\mathrm{~m}$ & GBM & none & 0 & 0.7 & 0.0 & - & - & - & a & 1.8 & 2.3 & $\mathrm{CR}$ & wt & methylated \\
\hline 39 & 59 & $\mathrm{~m}$ & GBM & none & 0 & 5.5 & 0.0 & - & - & - & $a, d$ & 1.9 & 3.0 & $\mathrm{CR}$ & wt & unmethylated \\
\hline 40 & 66 & $\mathrm{~m}$ & GBM & none & 7 & 56.4 & 0.0 & - & - & - & $a, d$ & 2.5 & 4.6 & $\mathrm{CR}$ & wt & unmethylated \\
\hline
\end{tabular}




\begin{tabular}{|c|c|c|c|c|c|c|c|c|c|c|c|c|c|c|c|c|}
\hline 41 & 65 & $\mathrm{~m}$ & GBM & none & 0 & 3.2 & 1.9 & 0.69 & 0.53 & $93 \%$ & $a$ & 2.4 & 4.3 & CR & wt & unmethylated \\
\hline 42 & 55 & $\mathrm{~m}$ & GBM & none & 0 & 22.0 & 0.0 & - & - & - & $a$ & 2.0 & 3.5 & B & wt & unmethylated \\
\hline 43 & 26 & $f$ & GBM & none & 13 & 3.5 & 0.0 & - & - & - & $a$ & 1.7 & 2.1 & CR & wt & methylated \\
\hline 44 & 33 & $\mathrm{~m}$ & GBM & none & 0 & 15.8 & 1.3 & 0.11 & 0.06 & $75 \%$ & $a$ & 2.0 & 3.2 & PR & mut & methylated \\
\hline 45 & 72 & $\mathrm{~m}$ & GBM & none & 8 & 4.8 & 4.4 & 0.67 & 0.50 & $70 \%$ & a & 2.2 & 3.6 & B & wt & unmethylated \\
\hline 46 & 57 & $\mathrm{~m}$ & GBM & none & 0 & 12.2 & 1.8 & 0.23 & 0.13 & $92 \%$ & $\mathrm{~b}$ & 2.3 & 5.0 & B & wt & unmethylated \\
\hline 47 & 78 & $f$ & GBM & none & 7 & 15.1 & 31.9 & 0.34 & 0.20 & $53 \%$ & $b$ & 1.8 & 2.6 & B & wt & unmethylated \\
\hline 48 & 57 & $f$ & GBM & none & 1 & 1.4 & 0.7 & 0.58 & 0.41 & $91 \%$ & - & 2.1 & 3.6 & B & wt & unmethylated \\
\hline 49 & 67 & $f$ & GBM & none & 9 & 29.7 & 0.0 & - & - & - & $b$ & 2.0 & 3.0 & $B$ & wt & unmethylated \\
\hline 50 & 44 & $\mathrm{~m}$ & GBM & none & 0 & 0.0 & 0.0 & - & - & - & $a$ & - & - & B & mut & methylated \\
\hline Mean & 56 & & & & 5 & 21.1 & 9.2 & 0.39 & 0.26 & $77 \%$ & & 2.2 & 4.2 & & & \\
\hline SD & 14 & & & & 7 & 14.5 & 11.2 & 0.21 & 0.16 & $26 \%$ & & 0.4 & 1.4 & & & \\
\hline Min & 26 & & & & 0 & 0.0 & 0.0 & 0.02 & 0.01 & $8 \%$ & & 1.2 & 3.8 & & & \\
\hline Max & 79 & & & & 30 & 56.4 & 44.6 & 0.70 & 0.54 & $100 \%$ & & 3.3 & 8.7 & & & \\
\hline
\end{tabular}

B: stereotactic biopsy; CR: complete resection; DSC: Dice similarity coefficient; EoR: extent of tumour resection; FET vs. FLAIR: a: FET < FLAIR; b: FET = FLAIR c: FET > FLAIR; d: FET partially outside of FLAIR; GBM: glioblastoma; IDH: isocitrate dehydrogenase; JSC: Jaccard similarity coefficient; MGMT: O6. methylguanine-DNA methyltransferase; mut: mutant; $\mathbf{n}$.a.: not available; OV: overlap volume; PR: partial resection; TBR mean: mean tumour-to-brain ratio; TBR max: maximum tumour-to-brain ratio; $\mathbf{V}_{\mathrm{FET}}$ : tumour volume defined by FET PET; $\mathbf{V}_{\mathrm{CE}}$ : tumour volume defined by contrast-enhanced MRI; wt = wildtyp 
Fig. 1 Differences between the Dice similarity coefficient (DSC), Jaccard similarity coefficient (JSC), and the overlap volume (OV). Similarity coefficients are commonly used for comparing algorithms for image segmentation against a known reference mask (ground truth). To find out the volumetric differences between two different imaging modalities, e.g., FET PET and contrast enhancement, the ground truth (true tumour extent) is usually not known. Thus, the results from similarity coefficients can be misleading and have to be interpreted with caution. (A) Two volumes of different size $(20 \mathrm{~mL} ; 10 \mathrm{~mL}$ ) are completely overlapping. (B) Two volumes of equal size (10 $\mathrm{mL}$ ) are only partially overlapping. Nevertheless, the similarity coefficients DSC and JSC yield identical results in both cases (DSC, 0.7; JSC, 0.5), indicating the same spatial similarity. On the other hand, the measure OV is depending on the volume and gives different results in both cases (A: OV, 100\%; B: OV, 70\%). Therefore, the combination of OV with DSC and JSC allows an improved interpretation of volumetric properties

Fig. 2 Contrast-enhanced (CE) MRI (left column), fluid-attenuated inversion recovery (FLAIR) MRI (middle column), and FET PET (right column) of representative patients without contrast enhancement (patients \#49, \#39). The tumour volume segmented by FET PET based on a tumour-to-brain ratio of more than 1.6 is projected onto MRI images (red contour). A considerable portion of FET PET tumour volume is located outside the FLAIR hyperintensity in patient \#49 (top row). In general, there is a considerable discrepancy between CE MRI, FLAIR and FET PET 
Fig. 3 Differences in volume between FET PET (red) and contrast-enhanced MRI (blue). In $20 \%$ of the patients, no contrast enhancement was present (in 10 of 50 patients). In $86 \%$ of the patients, the FET PET positive volume is considerably larger than the respective volume of the contrast enhancement in MRI (in 43 of 50 patients); ${ }^{*} \mathrm{P}<0.001$

Fig. 4 Contrast-enhanced (CE) MRI (left column), CE MRI with a projection of the tumour volume segmented by FET based on a tumour-to-brain ratio of 1.6 or more (second column, red contour), FET PET (third column) and a 3D reconstruction of the segmented tumour volumes based on CE MRI (last column, white) and FET PET (last column, red) of representative patients. Patient \#4: The volume of contrast enhancement is larger than the volume of FET uptake $(21.9 \mathrm{~mL}$ vs. $14.0 \mathrm{~mL})$. The area of FET uptake is located partially outside and beyond the area of contrast enhancement. The spatial similarity and the overlapping volume are low (DSC, 0.16; JSC, 0.09; OV, 20\%). Patient \#28: The volume of contrast enhancement is smaller than the FET uptake $(0.5 \mathrm{~mL}$ vs. $14.3 \mathrm{~mL})$. The contrast enhancing volume is mainly contained in the FET PET volume. Despite a high overlap volume (OV, 89\%), the spatial similarity is low (DSC, 0.06; JSC, 0.03). In both examples, only the combined interpretation of overlapping volumes and spatial similarity measures leads to a correct impression of the spatial orientation 